\title{
A Lys49-PLA 2 myotoxin of Bothrops asper triggers a rapid death of macrophages that involves autocrine purinergic receptor signaling
}

\author{
F Tonello ${ }^{*, 1}$, M Simonato ${ }^{1}$, A Aita ${ }^{2}$, P Pizzo ${ }^{2}$, J Fernández ${ }^{2,3}$, B Lomonte $^{3}$, JM Gutiérrez ${ }^{3}$ and C Montecucco ${ }^{2}$
}

Lys49-PLA $A_{2}$ myotoxins, an important component of various viperid snake venoms, are a class of PLA-homolog proteins deprived of catalytic activity. Similar to enzymatically active PLA (Asp49) and to other classes of myotoxins, they cause severe myonecrosis. Moreover, these toxins are used as tools to study skeletal muscle repair and regeneration, a process that can be very limited after snakebites. In this work, the cytotoxic effect of different myotoxins, Bothrops asper Lys49 and Asp49-PLA, Notechis scutatus notexin and Naja mossambica cardiotoxin, was evaluated on macrophages, cells that have a key role in muscle regeneration. Only the Lys49-myotoxin was found to trigger a rapid asynchronous death of mouse peritoneal macrophages and macrophagic cell lines through a process that involves ATP release, ATP-induced ATP release and that is inhibited by various purinergic receptor antagonists. ATP leakage is induced also at sublytical doses of the Lys49-myotoxin, it involves $\mathrm{Ca}^{2+}$ release from intracellular stores, and is reduced by inhibitors of VSOR and the maxi-anion channel. The toxin-induced cell death is different from that caused by high concentration of ATP and appears to be linked to localized purinergic signaling. Based on present findings, a mechanism of cell death is proposed that can be extended to other cytolytic proteins and peptides.

Cell Death and Disease (2012) 3, e343; doi:10.1038/cddis.2012.68; published online 5 July 2012

Subject Category: Neuroscience

Snakebite envenoming is a highly relevant and neglected health problem on a worldwide basis, and most cases of envenoming in Latin America are caused by snakes of the genus Bothrops. ${ }^{1}$ Bothrops venoms, like those of many other viperid snakes, cause complex pathophysiological alterations with prominent local (necrosis, hemorrhage, blistering and edema) and systemic effects (bleeding, coagulopathy, cardiovascular shock and renal failure). ${ }^{2,3}$ Local tissue damage leading to dermonecrosis and myonecrosis is particularly relevant, as it is frequently followed by poor tissue regeneration and permanent sequelae. ${ }^{4}$

Myotoxins are the main snake venom components causing tissue necrosis and, upon injection into higher animals, they cause irreversible damage on skeletal muscle fibers. They are basic proteins that can be classified into three main groups belonging to structurally distinct protein families: the 'small' myotoxins, the cardiotoxins and the PLA myotoxins. $^{5}$ The pathology caused by cardiotoxins and $\mathrm{PLA}_{2}$ myotoxins develops rapidly and it is associated with marked damage to the sarcolemma, whereas pathology associated with 'small' myotoxins has a more delayed onset and sarcolemma damage is not apparent. ${ }^{6}$ Among fast acting myotoxins, cobra cardiotoxins are basic three-finger toxins deprived of catalytic activity, they cause severe tissue necrosis and systolic heart arrest in snakebite victims through ill-known mechanisms that involve formation of membrane pores. ${ }^{7}$ The $\mathrm{PLA}_{2}$ myotoxins form the largest group and are divided into 'Asp49', which catalyze the hydrolysis of the ester bond in the sn-2 position of glycerol-phospholipids, and their enzymatically inactive homologs 'Lys49'. The mechanisms of myotoxicity of these toxins are largely unknown and, catalytically dependent and independent events are involved; in the case of the enzymatically inactive Lys49-PLA 2 homologs, an evident membrane disruption takes place without phospholipid hydrolysis. A common structural characteristic of locally acting myotoxins is a cationic face, which is likely to interact with negatively charged phospholipid bilayers. This initial electrostatic interaction, in the case of $B$. asper Mt-II, is probably followed by the penetration and disorganization of the membrane by the C-terminal region of the toxin. $2,5,8$

\footnotetext{
${ }^{1}$ Istituto di Neuroscienze - CNR Sezione di Padova, Italy; ${ }^{2}$ Dipartimento di Scienze Biomediche, Università di Padova, Italy and ${ }^{3}$ Instituto Clodomiro Picado, Facultad de Microbiología, Universida d de Costa Rica, San José, Costa Rica

*Corresponding author: F Tonello, CNR Sezione di Padova, Istituto di Neuroscienze, Viale G. Colombo, 3, Padova 35131, Italy. Tel: +39 049 8276057 ; Fax: +39049 8276049; E-mail: fiorella.tonello@cnr.it

Keywords: Lys49-PLA ; phospholipase $\mathrm{A}_{2}$; myotoxin; notexin; cardiotoxin; purinergic signaling

Abbreviations: BBG, Coomassie brilliant blue G; CBX, carbenoxolone; Ctx, Naja mossambica cardiotoxin; DIDS, 4,4'-Diisothiocyano-2,2'-stilbenedisulfonic acid; EGTA, ethylene glycol tetraacetic acid; FFA, flufenamic acid; GPCR, G-protein coupled receptor; iPLA2, calcium independent PLA2; LDH, lactic acid dehydrogenase; MFQ, mefloquine; Mt-I, B. asper myotoxin I; Mt-II, B. asper myotoxin II; NA, niflumic acid; Ntx, Notechis scutatus notexin; PLA2, phospholipase A2; PPADS, Pyridoxalphosphate-6-azophenyl-2',4'-disulfonic acid; RB2, reactive blue 2; SITS, 4-Acetamido-40-isothiocyanostil-bene-2,20-disulfonic acid; VSOR, volume-sensitive outwardly rectifying anion channel

Received 06.12.11; revised 25.4.12; accepted 30.4.12; Edited by A Verkhratsky
} 
In $B$. aspervenom, various myotoxins have been identified. One enzymatically active $\mathrm{PLA}_{2}$ myotoxin, Mt-I (alternative name Mt-III) (P20474), and three Lys49-PLA 2 myotoxins, Mt-II (P24605), Mt-IV (P0C616/Q9PRT7) and M1-3-3 (Q9PVE3), are presently reported in UNIPROT database, and together $\mathrm{PLA}_{2} \mathrm{~S}$ myotoxins account for $15-35 \%$ of B. asper venom proteins. ${ }^{9}$ Cardiotoxins and $\mathrm{PLA}_{2}$ myotoxins are used as tools to study the regeneration and maturation of mammalian skeletal muscle, ${ }^{10}$ a very complex process that involves multiple types of cells, among which macrophages have a major role. ${ }^{11}$ These cells are recruited already in the first phase of muscle regeneration, after acute muscle damage, and have the dual role of scavengers that phagocytose necrotic debris and of promoters of myogenic differentiation. ${ }^{11}$ For these reasons, it is relevant to evaluate whether myotoxins are cytotoxic also for macrophages, as this may have implications in the model of muscle injury by myotoxins and in the reparative and regenerative processes after snakebites.

Notexin, an Asp49-PLA 2 of Notechis scutatus (Ntx), and cardiotoxin of Naja mossambica (Ctx) are among the most frequently used myotoxins in the study of the muscle regeneration process. ${ }^{10}$ In this work, the activity of these toxins was compared with that of Mt-I and Mt-II, on mouse peritoneal macrophages and on three macrophagic cell lines (RAW264.7, J774.A1 and N13). Only Mt-II was found to induce a rapid death of these cells. Moreover, as in $\mathrm{C} 2 \mathrm{C} 12$ myotubes $B$. asper myotoxins induce a massive ATP release ${ }^{12}$ the extracellular concentration and the role of this molecule in macrophage death were analyzed. Mt-II was found to induce an initial ATP release, followed by an ATP-induced ATP release, that participates in the onset of a rapid and asynchronous cell burst. This is a novel type of cell death, quite different from that induced by a massive extracellular addition of ATP in J774.A1 and N13 mouse macrophagic cell lines. ${ }^{13,14}$

Based on these data, a two-step model of Mt-II-induced cytotoxicity is proposed, with an initial alteration of the plasma membrane associated with purinergic signaling followed by cytolysis due to the insertion of the toxin into the lipid bilayer.

\section{Results}

Mt-II, but not other myotoxins, is cytolytic for macrophages. Mt-I, Mt-II, Ntx and Ctx activities were evaluated on isolated peritoneal mouse macrophages. Figure 1a shows that, among the four myotoxins studied and compared here, only Mt-II, the Lys49 myotoxin devoid of $\mathrm{PLA}_{2}$ activity, displays a significant toxicity on these cells. Similar results were obtained with the mouse macrophages cell lines RAW264.7 and J774.A1 (Figure 1b).

Mt-II induces ATP release from macrophages. Mt-II induces a dose-dependent release of ATP from RAW264.7 and J774.A1 cells (Figure 2a), whereas Mt-I, Ntx and Ctx, even at concentrations of $6 \mu \mathrm{M}$, are without effect. ATP release was detected also at a sublytical concentration of Mt-II $(0.35 \mu \mathrm{M})$ with no significant LDH release, while at a higher toxin concentration extracellular ATP reaches its maximum level very rapidly, but this occurs always before the peak of $\mathrm{LDH}$ release (Figure $2 \mathrm{~b}$ ) and subsequently it decreases, presumably because of the action of ecto-ATP hydrolases (ecto-ATPases). In fact, in the presence of the ectoATPase inhibitor ARL67156 $(100 \mu \mathrm{M})$, and by sequestering calcium, a cofactor of ecto-ATPases, with EGTA, the extracellular ATP concentration keeps on increasing, together with that the extracellular LDH (Supplementary Figure S1). Altogether these experiments indicate that ATP release is not only due to a general plasma membrane damage but also because of a specific mechanism of ATP release induced by the toxin occurs in the initial 3-5 min after the intoxication.

The Mt-Il-induced ATP release from macrophages is inhibited by anionic channel antagonists and involves intracellular $\mathrm{Ca}^{2+}$ signaling. Different modes for ATP release have been proposed: via vesicle exocytosis, by the opening of channels, such as pannexins and connexins, or through specific anionic channels. ${ }^{15,16}$ To investigate the possible pathways for ATP release, RAW264.7 and J774.A1 cells were exposed to Mt-II in the presence of bafilomicyn A1, which prevents ATP exocytosis by inhibiting the v-ATPase proton pump, or in the presence of inhibitors of various potential ATP channels. ATP release was measured after $5 \mathrm{~min}$, at the time of maximum concentration of released ATP and before important percentage of cell lysis, as indicated by the kinetics of LDH release (Figure 2).

Figure 3A shows that neither bafilomycin A1 nor connexin and pannexin inhibitors (carbenoxolone, flufenamic acid,
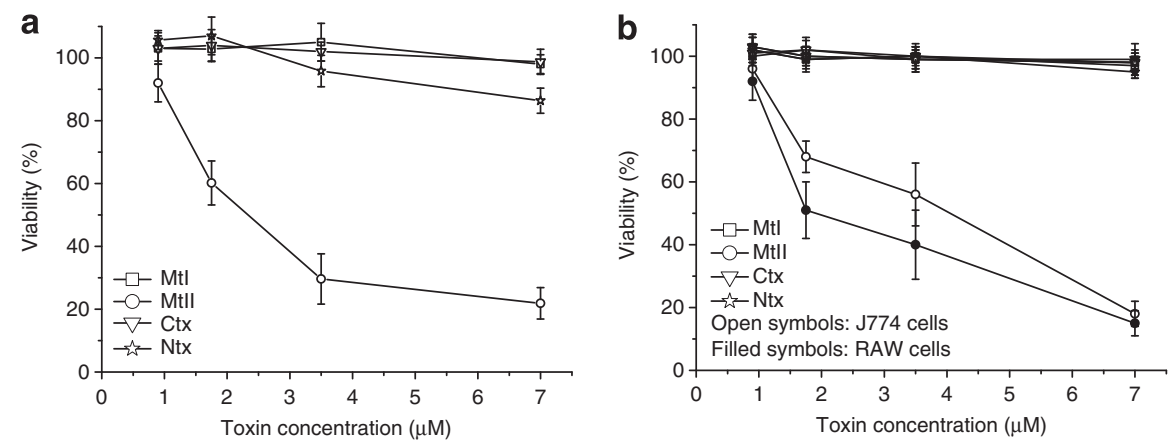

Figure 1 Cell death induced by Mt-I, Mt-II, Ctx and Ntx. Cytotoxicity was measured with the MTS assay on mouse peritoneal macrophages (a) and on macrophagic cell lines RAW264.7 and J774.A1 (b) as a function of the toxin concentration in the medium. Macrophages were incubated with the different toxins in the mKRB medium (see Materials and Methods section) for $1 \mathrm{~h}$, and cell viability was determined. Values are mean \pm S.D.; $n=4-5$ 
a

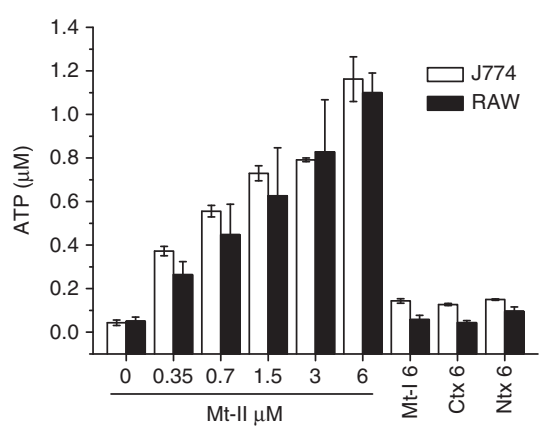

b
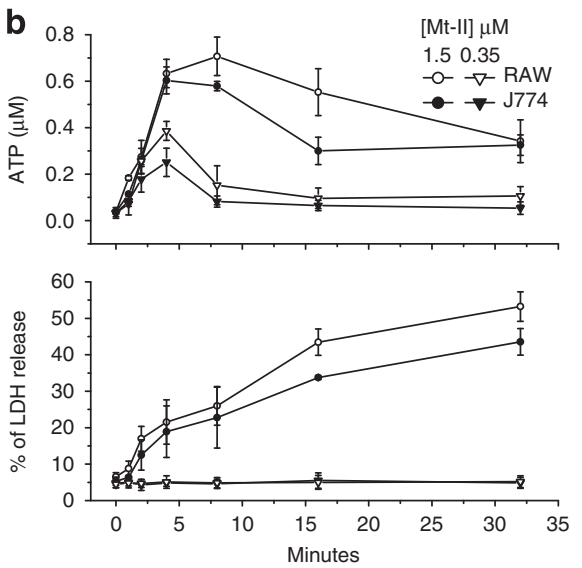

Figure 2 ATP release induced by Mt-I, Mt-II, Ctx and Ntx. (a) Mt-II induces a dose-dependent release of ATP from RAW264.7 and J774.A1 cells. Mt-I, Ctx and Ntx (6 $\mu$ M) do not induce a significant release of ATP. ATP was measured 5 min after intoxication with a luciferin/luciferase-based assay. (b) Kinetics of ATP (top panel) and LDH (bottom panel) release from J774.A1 and RAW264.7 cells induced by 0.35 and $1.5 \mu \mathrm{M} \mathrm{Mt-II.} \mathrm{Notice} \mathrm{that} \mathrm{ATP} \mathrm{release} \mathrm{temporally} \mathrm{precedes} \mathrm{that} \mathrm{of} \mathrm{LDH.} \mathrm{Values} \mathrm{are} \mathrm{mean} \pm$ S.D.; $n=4$

mefloquine and niflumic acid) reduced the ATP release. In contrast, antagonists of the maxi-anion channel and of VSOR inhibited the process, indicating that these channels are involved. SITS and DIDS inhibit both the maxi-anion channel and VSOR, whereas phloretin is specific for VSOR and gadolinium ion is specific for the maxi-anion channel. ${ }^{17,18}$

As ATP release is elicited by stimulation of receptors that induce cytosolic calcium increase, ${ }^{15}$ the role of this ion in the mechanism of Mt-II-induced ATP release was investigated. Mt-II was found to cause an increase in cytosolic $\left[\mathrm{Ca}^{2+}\right]$, as observed by calcium imaging of cells loaded with fura-2, both in the presence or absence of external $\mathrm{Ca}^{2+}$, indicating that this toxin triggers a release of $\mathrm{Ca}^{2+}$ from intracellular stores (Figure 3B, left panels). This was confirmed by depleting the intracellular $\mathrm{Ca}^{2+}$ stores by pre-treating cells with cyclopiazonic acid and EGTA, and observing that in this case the toxin does not induce an increase in cytosolic $\left[\mathrm{Ca}^{2+}\right]$ (Figure 3B, bottom-right panel).

A link between calcium signaling and ATP release was established by preloading cells with BAPTA-AM, a chelator of cytosolic $\mathrm{Ca}^{2+}$, or by exposing cells to EGTA-containing medium, before intoxication with Mt-II. BAPTA-AM, but not EGTA addition, reduced the released ATP, 5 min after intoxication, to about $50 \%$, confirming that the calcium signal mediating this process is predominantly derived from intracellular stores (Figure $3 \mathrm{C}$ ).

Mt-Il-induced cell death is inhibited by ATP anion channel and purinergic receptor antagonists. The same compounds that inhibit the Mt-II-induced ATP release, protect cells from death due to the intoxication (Table 1, lines 1-9). Also BAPTA confers some protection from Mt-II intoxication, whereas EGTA is not protective (Table 1, lines 10-11). The role of purinergic receptors was then investigated by evaluating the protective action of $P 2 X$ and $P 2 Y$ antagonists on Mt-II-induced cell death. P2Xs are ligandgated cation channels activated by extracellular ATP, whereas P2Ys are G-protein coupled receptors activated by ATP, ADP, UTP, UDP and UDP-glucose. To date, seven members of the $P 2 X$ family $(P 2 \times 1-7)$ and eight $P 2 Y$ receptors (P2Y1, P2Y2, P2Y4, P2Y6, P2Y11, P2Y12, P2Y13 and P2Y14) have been characterized. ${ }^{19}$ Upon activation, these receptors, which are widely distributed throughout the body, modulate an array of cellular functions, such as plasma membrane permeabilization, $\mathrm{Ca}^{2+}$ influx and cell death. ${ }^{19}$ Expression profiles of all these receptors, except P2Y11, have been verified in RAW264.7 cells $^{20}$ (Supplementary Figure S2).

Table 1 (lanes 12-24) reports the protection, of macrophage cell death induced by Mt-II, obtained with various inhibitors of purinergic receptors, as well as the concentration and the role of the inhibitors. From the pattern of inhibitors showing a protective role, it appears that more than one type of membrane purinergic receptor/channel is involved in this cell death process. A prominent role appears to be played by P2 $\times 1,2$ or 3 (inhibited by NF449, NF023, PPADS, BBG, RB2) and by P2Y12 and 13 (selectively inhibited by MRS2211 and clopidogrel). However, the inhibition by OATP also involves $\mathrm{P} 2 \times 7$. P2Y1 and P2 $\times 4$ may not have an important role as their specific inhibitors, MRS2500 and 5-BDBD, respectively, are not effective. $\mathrm{P} 2 \mathrm{Y} 6$, the purinergic receptor expressed at the highest level in RAW264.7 cells $^{20}$ (Supplementary Figure S2), is inhibited by MRS2578 that is not fully protective against Mt-II cytotoxicity. Inhibitors of the signal transduction pathways, activated by P2Ys and by GPCRs, are also protective (bottom lines of Table 1), including BEL, an inhibitor of the cytosolic $\mathrm{Ca}^{2+}$-independent phospholipases. Importantly, some of these purinergic antagonists inhibit the ATP release induced by the toxin (Figure 4) indicating that the first released ATP may induce a further release of the same molecule, triggering a positive feedback.

Mt-Il-induced cell death differs from that induced by high ATP concentration. High extracellular ATP concentration is toxic for several cells including the J774.A1 macrophage cell line. ${ }^{14}$ So the death process induced by ATP in J774.A1 macrophages and in N13 microglial cells, another cell line sensitive to ATP, ${ }^{13}$ was compared with the death induced by Mt-II intoxication. N13 are as sensitive to Mt-II intoxication as 

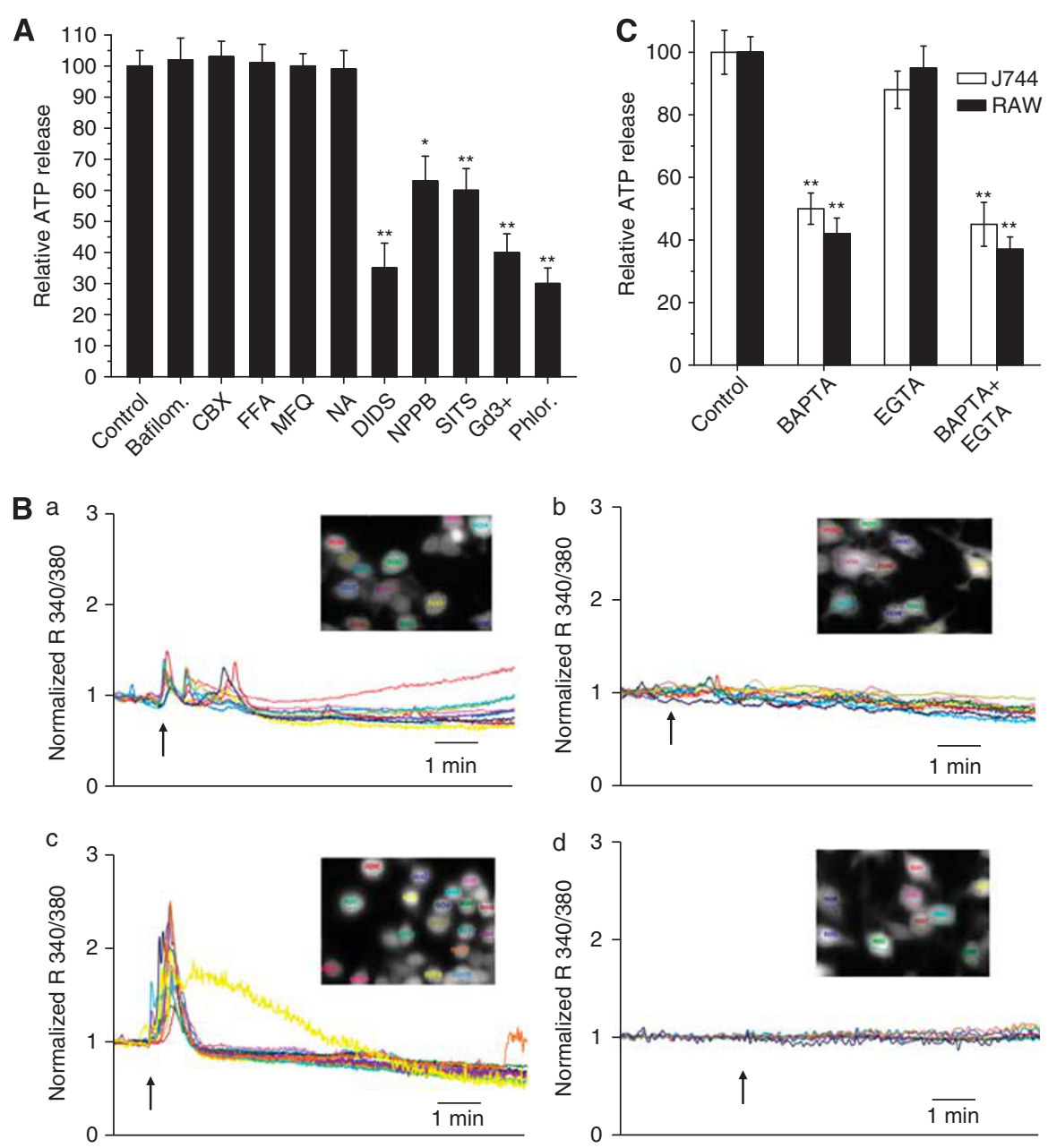

Figure 3 Characterization of the ATP release induced by Mt-II $(1.5 \mu \mathrm{M})$ on macrophages. (A) Effect of bafilomycin and inhibitors of ATP channels. ATP present in the extracellular medium was determined 5 min after the intoxication. Values are mean \pm S.D. of four independent experiments on J774.A1. RAW264.7 cells give identical results (data not shown). Statistically significant differences were determined via an one-tailed Student's $t$-test $\left({ }^{*} P<0.01 ;{ }^{* \star} P<0.001\right)$. Inhibitors were used at the same concentrations indicated in Table 1. Bafilomycin A1 was used at $0.2 \mu \mathrm{M}$. (B) Calcium imaging of RAW264.7 cells loaded with fura-2 AM and treated with Mt-II (1.5 $\mu \mathrm{M})$ in mKRB (top-left panel) or in $\mathrm{Ca}^{2+}$-free EGTA $(0.2 \mathrm{mM}$ )-containing medium (bottom-left panel). Top- right panel shows an addition of the same volume of mKRB without the toxin to fura-2 loaded cells. Bottom-right panel: fura-2 loaded cells were pretreated, before the intoxication with Mt-II in mKRB, with cyclopiazonic acid (20 $\mu$ M, CPA) and EGTA $(0.6 \mathrm{mM})$ to deplete intracellular calcium stores. The arrows indicate the time at which the toxin was added. The same results were obtained in at least three independent experiments for each condition. The inserts correspond to pictures of the evaluated cells before the addition of toxin or buffer, to correlate each colored trace to a specific cell. Representative ratios from each picture are shown. Similar results were obtained with $\mathrm{J} 774$ cells (data not shown). (C) Release of ATP induced by Mt-II, 5 min after the intoxication, in cells preloaded with BAPTA-AM $(2 \mu \mathrm{M})$ or in the presence of EGTA $(1.5 \mathrm{mM})$ in the extracellular medium. Values are mean \pm S.D. of four independent experiments. Statistically significant differences were determined via an one-tailed Student's $t$-test $\left({ }^{\star} P<0.01 ;{ }^{* \star} P<0.001\right)$

macrophages (Supplementary Figure S3), with a similar, though not identical, profile of activity of the array of inhibitors used in the previous section (Supplementary Table S1). When RAW264.7, J774.A1 or N13 cells are intoxicated with Mt-II, lysis occurs in a sudden but not synchronized way: cells undergo volume variations, swelling and shrinkage, some of them form large plasma membrane blebs with exposure of phosphatidylserine, and eventually cells burst with a random distribution (Figure 5, Supplementaty Videos S1 and S2). In contrast, J774.A1 and N13 cells treated with high ATP concentration form little and diffuse membrane blebs and die in a slower synchronized way (Supplementary Video S3). A time to death of $6 \mathrm{~h}$ was reported in cells exposed to very high concentration of ATP $(>3 \mathrm{mM}),{ }^{13,14}$ whereas at the dose of the toxin $(1.5 \mu \mathrm{M})$ used in the present work $50 \%$ of the cells dies in less than $1 \mathrm{~h}$. Moreover, also the changes of cytosolic $\left[\mathrm{Ca}^{2+}\right]$ in $\mathrm{N} 13$ and J774.A1 cells, induced by high concentration of Mt-II and ATP, were found to be different (see Figure S4 and Figure S5 in Supplementary Materials, Video S4 and Video S5).

Apyrase from potato, an enzyme mixture that hydrolyzes nucleoside triphosphates and diphosphates, completely protect cells from death induced by ATP (data not shown), whereas it displays only a partial protection of cells exposed to Mt-II (Figure 6a). Moreover, the addition of ATP and ADP does not amplify the death induced by the toxin (Figure 6b). The lack of protection by apyrase can be due to the transformation of ATP into other toxic products as the final product of ATP degradation by apyrase is AMP, which is subsequently transformed into adenosine by extracellular 
Table 1 Protection percentage (Prot \%) of macrophagic cells, from Mt-IIinduced cytotoxicity, by inhibitors of ATP channels (first white area), calcium chelators (first shaded area), inhibitors of the purinergic receptors (second white area) and of intracellular signaling activated by GPCRs and/or purinergic receptors (second shaded area)

\begin{tabular}{|c|c|c|c|c|}
\hline Inhibitor & {$[\mu \mathrm{M}]$} & Target $^{a}$ & Prot $\%$ & S.D. \\
\hline Flufenamic acid & 10 & Pannexins and connexins & 0 & \\
\hline Niflumic acid & 10 & & 0 & \\
\hline Carbenoxolone & 100 & & 0 & \\
\hline Mefloquine & 30 & & 0 & \\
\hline DIDS & 30 & Maxi-anion channels and VSOR & 82 & $5^{\star *}$ \\
\hline NPPB & 100 & & 15 & $7^{*}$ \\
\hline SITS & 100 & & 31 & $2^{\star \star}$ \\
\hline $\mathrm{Gd} 3+$ & 50 & Maxi-anion chan.s & 94 & $4^{\star \star}$ \\
\hline Phloretin & 100 & VSOR & 93 & $5^{\star \star}$ \\
\hline BAPTA-AM & 2 & Intracell. $\mathrm{Ca}^{2+}$ chelator & 58 & $10^{\star *}$ \\
\hline EGTA & 1500 & Extracell. Ca chelator & 0 & \\
\hline O-ATP & 300 & $\mathrm{P} 2 \times 7$ & 65 & $4^{\star \star}$ \\
\hline 5-BDBD & 30 & $\mathrm{P} 2 \times 4$ & 0 & \\
\hline Suramin & 10 & P2X, P2Y; $\mathrm{G}_{2}$ & 100 & $2^{* \star *}$ \\
\hline NF449 & 10 & $\mathrm{P} 2 \times 1,1+5,2+3,3,2,4 ; \mathrm{Gs}_{\alpha}$ & 99 & $2^{\star \star \star}$ \\
\hline NF023 & 20 & $\mathrm{P} 2 \times 1,3,2,4 ; \mathrm{Go} / \mathrm{i}_{\alpha}$ & 72 & $7^{\star \star \star}$ \\
\hline PPADS & 10 & $\mathrm{P} 2 \times 1,2,3,5 ; \mathrm{P} 2 \mathrm{Y} 2,4$ & 99 & $2^{\star \star \star}$ \\
\hline BBG & 20 & $\mathrm{P} 2 \times 7,2,4,1,3$ & 98 & $4^{\star \star \star}$ \\
\hline RB2 & 20 & $\mathrm{P} 2 \times 2,1,2+3 ; \mathrm{P} 2 \mathrm{Y} 12,1,6$ & 97 & $3^{\star \star \star}$ \\
\hline Clopidogrel & 20 & P2Y12,13,GRP17 & 65 & $4^{\star \star}$ \\
\hline MRS2211 & 30 & $\mathrm{P} 2 \mathrm{Y} 13,1,12$ & 98 & $3^{\star \star \star}$ \\
\hline MRS2500 & 30 & P2Y1 & 0 & \\
\hline MRS2395 & 30 & $\mathrm{P} 2 \mathrm{Y} 1>>\mathrm{P} 2 \mathrm{Y} 13$ & 0 & \\
\hline MRS2578 & 20 & $\mathrm{P} 2 \mathrm{Y} 6>>\mathrm{P} 2 \mathrm{Y} 1,2,4,11$ & 26 & $16^{*}$ \\
\hline Gallein & 10 & $\mathrm{G} \beta \gamma$ & 79 & $4^{\star *}$ \\
\hline Pertussis toxin & 0.1 & $\mathrm{Gi}, \mathrm{G}_{0}, \mathrm{G}_{\mathrm{t}}$ & 45 & $10^{\star *}$ \\
\hline Neomycin & 100 & PLC & 29 & $11^{*}$ \\
\hline BEL & 10 & iPLA2 & 63 & $12^{* *}$ \\
\hline
\end{tabular}

Inhibitor concentrations non-toxic to cells were used. Data reported are the average values obtained with a minimum of four independent experiments on RAW264.7 and J774.A1 cells. Statistically significant differences were determined via an one-tailed Student's $t$-test $\left({ }^{\star} P<0.05 ;{ }^{\star \star} P<0.01\right.$ $\left.{ }^{\star \star *} P<0.001\right)$

${ }^{\text {ap }}$ urinergic receptors are listed in order of decreasing sensitivity for the antagonist

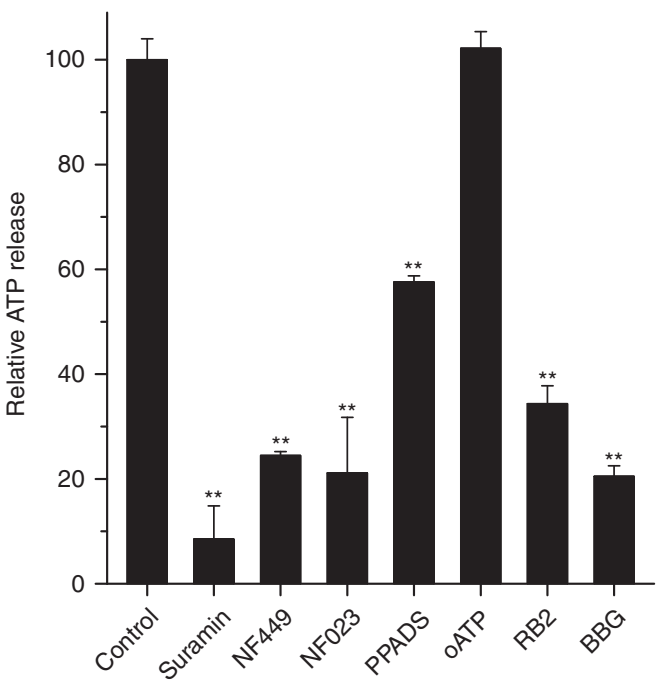

Figure 4 Effect of some purinergic inhibitors on the ATP release induced by Mt-II in J774.A1 cells. Experiments were performed as reported in Figure 3A. All inhibitors were pre-incubated for $30 \mathrm{~min}$. Statistically significant differences were determined via a one-tailed Student's $t$ test $\left({ }^{*} P<0.001\right)$

5 '-nucleotidases. Adenosine is present in many snake venoms and it has been proposed to have a role in envenoming effects. ${ }^{21}$ To test whether adenosine also participates in the cell death process, the effects of caffeine, a generic adenosine receptor inhibitor, and of adenosine itself, were evaluated. The results shown in Figure $6 \mathrm{~b}$ indicate that adenosine receptors are not involved in the Mt-II-induced cell death mechanism.

\section{Discussion}

The results reported here show that Mt-II, a catalytically inactive $\mathrm{PLA}_{2}$-homolog myotoxin, induces an ATP release from mouse macrophages that is involved in a death process through an ATP positive feedback mechanism. This toxin has been reported to cause activation of some functions, that is, phagocytosis and respiratory burst, in mouse peritoneal macrophages at sublytic concentrations. ${ }^{22}$ The physiological consequences of all these effects, and of the cytotoxicity, in vivo remain to be elucidated. In skeletal muscle, there is a population of resident macrophages, ${ }^{23}$ which are likely to be affected by these toxins following envenomation. In addition, infiltration of the tissue by macrophages follows the process of myonecrosis. Initially, a population of 'inflammatory' macrophages predominates, with a role in removal of necrotic debris and, subsequently, a population of 'non-inflammatory' macrophages appears, releasing factors required for skeletal muscle regeneration. ${ }^{11}$ It is suggested that, upon venom injection, myotoxins interact with resident macrophages, inducing activation and cytotoxicity. As the inflammatory population of macrophages appears many hours after the onset of myonecrosis, it is possible that the toxin concentration in the tissue, by the time these macrophages reach the affected muscle is low. However, owing to the leading role that macrophages have in the process of muscle regeneration, the possible effect of myotoxins in these cells in vivo warrants further investigations.

In contrast to Mt-II, Mt-I, Ntx and Ctx are not cytolytic for macrophages and do not induce a significant release of ATP from these cells. These results suggest that Mt-II is likely to have an acceptor in macrophages, which mediates the observed effects, and given the wide cytotoxic profile of $\mathrm{Mt}-\mathrm{II},{ }^{24}$ this receptor is likely to be ubiquitous. Mt-I, Ntx and Ctx either do not have an acceptor molecule in macrophages or their binding does not result in ATP release and cytotoxicity. Mt-I, despite the lack of cytotoxic effect on macrophages, induces signaling activation and lipid body formation in these cells $^{25}$ and ATP release and a rapid cell lysis in $\mathrm{C} 2 \mathrm{C} 12$ myotubes. ${ }^{12}$ Therefore, it is possible that the Mt-I receptor(s) is present in both macrophages and $\mathrm{C} 2 \mathrm{C} 12$ but induces different signaling in the two cell types, or the Mt-I receptor is present only in $\mathrm{C} 2 \mathrm{C} 12$ and the Mt-I effects in macrophages are due to the $\mathrm{PLA}_{2}$ enzymatic activity.

Present findings on ATP release and cytotoxicity data suggest that, among the possible ways of ATP release, Mt-II induces the opening of maxi-anion channel and VSOR, two anion channels still not identified at molecular levels, that are ubiquitous and involved in many pathological processes. ${ }^{17,18}$ These channels are known to be activated by cell swelling, ischemia and hypoxia and by various stimuli acting on GPCRs. Observations on $\mathrm{Ca}^{2+}$ release from intracellular stores induced by $\mathrm{Mt}$-II, indicate a possible involvement of GPCRs in toxin-induced ATP release, via activation of PLC and IP3 production; moreover, various animal toxins, and 

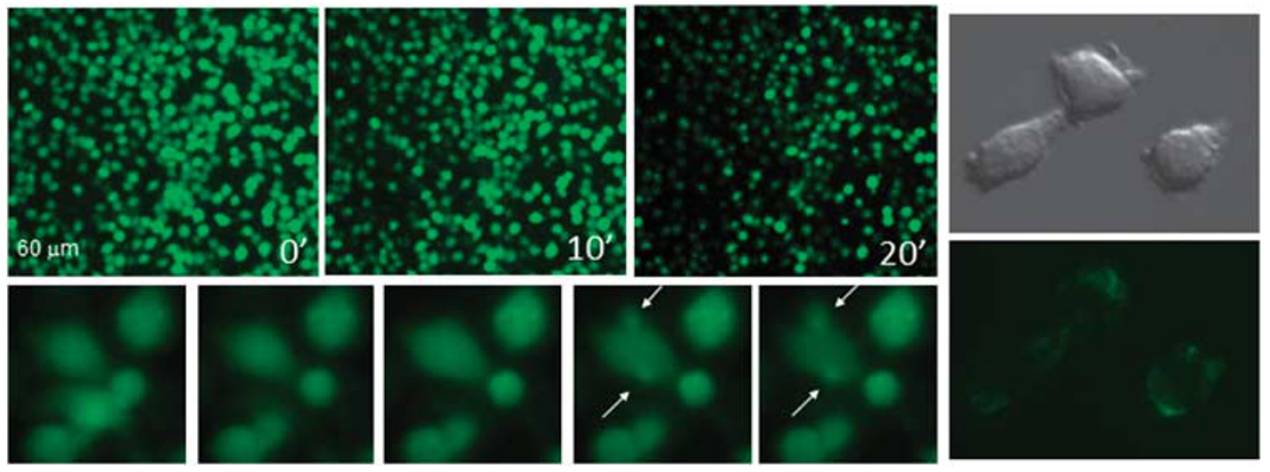

Figure 5 Observation by fluorescence microscopy of macrophage death induced by Mt-II (1.5 $\mu \mathrm{M})$. Left: J744.A1 cells were loaded with the fluorescent dye calcein and intoxicated with Mt-II. Top panels: cell death appears as a rapid burst of cells in an asynchronous, 'spotted-like' way (see also Supplementaty Video S1). Bottom panels: some cells swell and form characteristic membrane blebs (arrows). Images have been recorded with 10" time intervals (see also Supplementaty Video S2). Right panels: RAW264.7 cells intoxicated with Mt-II in the presence of annexin-V-fluorescein. Membrane blebs induced by Mt-II intoxication show surface exposure of phosphatidylserine (see also Supplementary Figures S6 A and B). Cell integrity was checked by staining with propidium iodide. All experiments were repeated at least three times
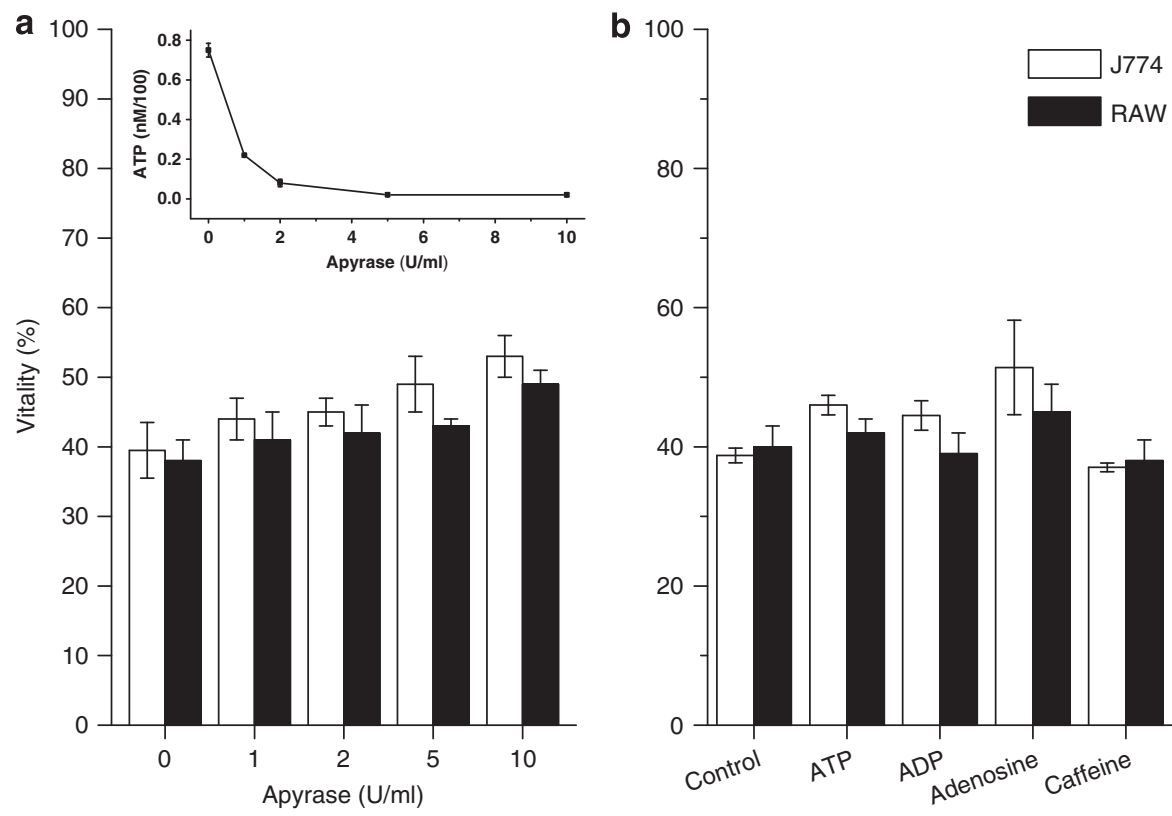

Figure 6 Effect of apyrase (a), of various purine derivatives (100 $\mu \mathrm{M}$ each) and of caffeine $(10 \mu \mathrm{M})(\mathbf{b})$ on Mt-II-induced cytotoxicity. Apyrase activity was checked by determining ATP in the extracellular medium of J744.A1 cells incubated with Mt-II (1.5 $\mu \mathrm{M}$, inset). Values are mean \pm S.D. of four independent experiments on J774.A1 and RAW264.7 cells

among them also some $\mathrm{PLA}_{2} \mathrm{~S}$, have been found to bind GPCRs. ${ }^{26}$

Another possible mechanism for ATP release is the opening of $\mathrm{P} 2 \times 7$ channels, ${ }^{16}$ but the fact that the specific inhibitor, OATP, does not inhibit the ATP release argues against this possibility.

Mt-Il-induced cell death process appears to involve many purinergic receptors and also ectonucleotidases that transform ATP into ADP, agonist of the P2Y1, 2, 12 and 13 receptors. Precise information on the receptors involved is difficult to obtain using only a pharmacological approach, as some drugs may affect more than one target and some of them such as suramin but probably also its analog NF449, also directly interact with Mt-II. ${ }^{27}$ Nonetheless, from the present pharmacological inhibition profile, $\mathrm{P} 2 \times 1-3$ and P2Y12-13 appear to be main players in the Mt-II-induced macrophage death process. In addition, other receptors that could be involved are P2 $\times 7$, P2Y2 and P2Y6. Purinergic receptors seem to be involved also in a Mt-II-induced, positive feedback mechanism of ATP release, as purinergic inhibitors reduce the amount of released ATP. The alternative possibility that all these inhibitors block the supposed myotoxin receptor is very unlikely, as it would imply that molecules of different structure bind to the same target. Moreover, from previous data, it clearly appears that purinergic receptors, in different cell types, are often involved in positive feedback mechanisms: for example, the P2 $\times 2$ receptor seems to be necessary for an autocrine positive feedback signal amplifying taste-evoked ATP secretion, ${ }^{28}$ whereas $\mathrm{P} 2 \times 3$ constitutes a positive autocrine signal for insulin release in human pancreatic beta cells. ${ }^{29}$ Moreover, P2Y12 together with P2Y2 and adenosine receptors are 
involved in autocrine purinergic feedback loops induced by the chemoattractant $\mathrm{C} 5 \mathrm{a}$, in macrophages, through the release of ATP. ${ }^{30}$

Besides $\mathrm{Mt}^{12}{ }^{12}$ and $\mathrm{Mt}-\mathrm{Il}$, other membrane-perturbing polypeptides have been reported to stimulate ATP efflux. Some antimicrobial peptides were found to stimulate ATP release from Candida albicans and the released ATP was suggested to participate in the lytic process by an unknown mechanism. ${ }^{31}$ Alpha-hemolysin, a pore-forming toxin, was shown to involve purinergic signaling via $\mathrm{P} 2 \mathrm{X}$ receptors and pannexins, ${ }^{32}$ and to stimulate surface exposure of phosphatidylserine. ${ }^{33}$

The current model for the Mt-II cytolytic mechanism posits that this toxin inserts in the plasma membrane and perturbs its permeability properties. ${ }^{5}$ Moreover, it is known that the presence of anionic lipids ${ }^{34,35}$ and the membrane fluidity ${ }^{36}$ affect the cytolytic activity of Mt-II and other myotoxins, that is, human red blood cells, insensitive to Mt-II, become susceptible to the toxin after enrichment with phosphatidylserine, ${ }^{34}$ whereas cell membrane cholesterol depletion, by methyl- $\beta$ cyclodextrin treatment, enhanced the Mt-II cytolytic action on C2C12. ${ }^{36}$

Taking together previous and present results, it is suggested that purinergic signaling activation by Mt-II alters the bilayer composition and the asymmetry of the plasma membrane and makes it more susceptible to the lytic action of the toxin. This is consistent with the finding that purinergic signaling activation is involved in membrane ruffling and pseudopod protrusion during immune cell chemotaxis, ${ }^{30,37}$ a process that is mediated also by intracellular phospholipases, ${ }^{38}$ whereas phosphatidylserine distribution and membrane blebbing are known to involve P2 $\times 7$ and iPLA2 activation. $^{39}$ Moreover, the $\mathrm{P} 2 \mathrm{Y} 2$ and other purinergic receptors are involved in ATP-mediated autocrine and paracrine mechanisms stimulated by HIV-1, facilitating fusion between viral and cell membranes. ${ }^{40}$

The random distribution of the Mt-II-induced cell lysis indicates that the product(s) released by a dying cell does not facilitate the death of neighboring cells. This observation, together with the lack of a significant protective effect of apyrase and of a synergism between ATP or ADP addition and the Mt-II-induced cell death, suggests that the process activated by the myotoxin is probably localized in cell membrane microdomains where ATP is released, by the opening of specific channels, and rapidly intercepted by local ectonucleotidases and purinergic receptors. Purinergic receptor compartmentalization has been observed in different cells. ${ }^{19}$ Moreover, the maxi-anion channels in adult cardiomyocytes are localized at the opening of T-tubules and along Z-lines ${ }^{17}$ and this distribution may be relevant to the activity of Mt-II on myotubes. ${ }^{12}$

In conclusion, the protection from Mt-II, achieved in macrophagic cells by various inhibitors of purinergic signaling, suggests a novel two-step mechanism for the toxin action. In the first step, cytotoxins interact with and activate, already at low concentrations, a specific receptor, in a membrane microdomain, that triggers a signaling process, which alters the membrane composition and properties. This membrane alteration renders the bilayer more susceptible to the action of Mt-II. Then, in the second step, the toxin molecules insert into

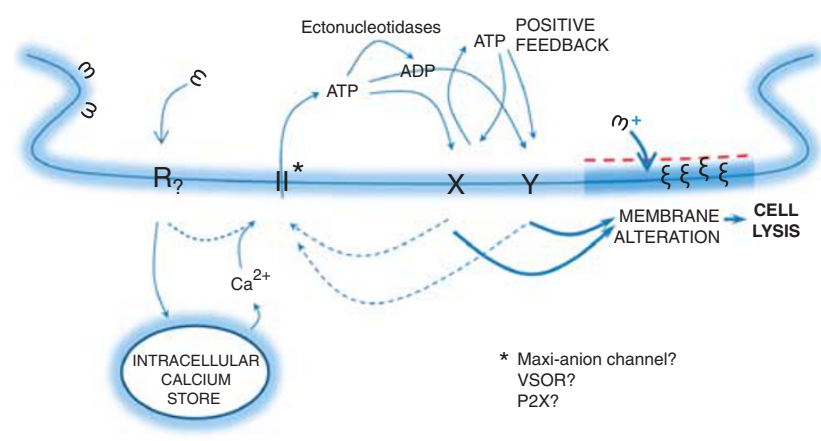

Figure 7 Model of action of Mt-II. The toxin interacts with a yet unidentified receptor $(R)$, embedded in a membrane microdomain. $R$ activates ATP channels by inducing the release of calcium from intracellular stores and/or by other mechanisms. The released ATP stimulates purinergic receptors within the same plasma membrane microdomain inducing a further release of ATP. The extracellular ATP activates a signaling cascade leading to changes in the composition of the plasma membrane, which in turn promotes the insertion of Mt-II into the lipid bilayer with consequent osmotic cell lysis. It is proposed that this hypothesis may be valid also for other membrane-active toxins and peptides

the altered membrane and, if present at high concentration, cause a rapid cell lysis (Figure 7). Considering that other cytolysins have been found to induce ATP release, this hypothetic mechanism of action may be extended to other toxins, which act by changing the plasma membrane permeability and suggests that the possible involvement of purinergic mechanisms should be tested. The involvement of a receptor that induces the ATP release can explain the cell specificity of these membrane perturbing toxins although an interaction with specific cell membrane lipids cannot be excluded.

Finally, the purinergic receptor inhibitors and other compounds, which were found here to be protective in the Mt-II cytotoxicity assay should be evaluated as possible therapeutics for ameliorating the tissue damage induced by snake venoms.

\section{Materials and Methods}

Materials. Myotoxins I and II were isolated from the crude venom of Bothrops asper, a pool obtained from at least 30 specimens kept at the serpentarium of Instituto Clodomiro Picado, University of Costa Rica, as described before. ${ }^{12}$ Notexin from Notechis scutatus was purchased from Latoxan (Valence, France) and Naja mossambica cardiotoxin from Sigma. All inhibitors were purchased from Tocris or Sigma (Milan, Italy). Pertussis toxin was obtained from Tocris (Abingdon, UK) and potato apyrase from Sigma (A7646). BAPTA-AM and calcein were obtained from Life Technologies (Monza, Italy).

Cell cultures. The murine macrophage cell lines J774.A1 (ATCC TIB 67) and Raw264.7 (ATCC TIB 71) were obtained from the American Type Culture Collection and maintained in Dulbecco's modified Eagle's medium supplemented with heat-inactivated fetal bovine serum (EuroClone, Milan, Italy) $10 \%, 10 \mu \mathrm{g} / \mathrm{ml}$ streptomycin, $10 \mathrm{U} / \mathrm{ml}$ penicillin. The microglial $\mathrm{N} 13$ cell line $^{13}$ was cultured in RPMI 1640 medium supplemented with $2 \mathrm{mM}$ glutamine, $10 \%$ heat-inactivated FBS, $10 \mu \mathrm{g} / \mathrm{ml}$ streptomycin, $10 \mathrm{U} / \mathrm{ml}$ penicillin.

Primary macrophages were isolated from the peritoneum of CD-1 mice. All experimental procedures were carried out in accordance to the European Community Council Directive no. 86/609/EEC and were approved by the local authority veterinary service, project number 24088/ 2011. Mice were intraperitoneally injected with thioglycollate broth. After 3-5 days, animals were killed under $\mathrm{CO}_{2}$ atmosphere, peritoneal cavities were washed with $3 \mathrm{ml}$ of phosphate buffer solution and the fluid was collected, centrifuged at $1200 \times \mathrm{g}$, and the cell sediment was resuspended in a complete medium (RPMl added with $10 \%$ fetal calf 
serum). Cells were seeded in 96-well plates and after adhesion, cultures were washed three times with phosphate buffer solution to remove non-adherent cells. More than $95 \%$ of the cell population consisted of macrophages, as determined by conventional morphological criteria.

Cytotoxicity assay. Cells seeded the day before in 96-well plates (40000/ well) were washed once in modified Krebs-Ringer buffer (mKRB) $(140 \mathrm{mM} \mathrm{NaCl}$, $2.8 \mathrm{mM} \mathrm{KCl}_{2} 2 \mathrm{mM} \mathrm{MgCl}_{2}, 1 \mathrm{mM} \mathrm{CaCl}_{2}, 10 \mathrm{mM}$ Hepes and $11 \mathrm{mM}$ glucose, $\mathrm{pH} 7.4$ ) and incubated with Mt-II, diluted in mKRB (final volume $100 \mu \mathrm{l}$ ), for the indicated time or, in pharmacological tests, for the time necessary to obtain a $50 \%$ of death in the control samples (in presence of toxin and without inhibitors). Purinergic inhibitors, channel inhibitors and apyrase were added at the same time as Mt-II. Pertussis toxin was pre-incubated for $2 \mathrm{~h}$ in full cell medium. Gallein, BEL, forskolin and neomycin were pre-incubated for $30 \mathrm{~min}$ in mKRB. All inhibitors were checked for their effect on cell viability and used at non-toxic concentrations. Cell viability was measured with the MTS (3-(4,5-dimethylthiazol-2-yl-5-(3-carboxymethoxyphenyl-2-(4-sulfonphenyl)-2H-tetrazolium, inner salt) assay. The CellTiter 96 AQueous One Solution Cell Proliferation Assay (Promega, Milan, Italy) was used and instructions from the manufacturer were followed. The release of LDH was measured as an index of cellular necrosis using the commercial kit TOX7 (Sigma), which is based on the LDH-catalyzed reduction of $\mathrm{NAD}^{+}$, which then converts a tetrazolium dye to a soluble colored formazan derivative.

Protection of macrophage cells from Mt-II-induced cytotoxicity by pharmacological inhibitors was evaluated with both kinds of tests (cell viability and LDH release) with similar results. Results reported in Table 1 have been obtained with $\mathrm{LDH}$ release test.

ATP measurement. Cells were seeded, the day before, in 24-well plates, 150000 for each well. The growth medium was replaced with $225 \mu \mathrm{l}$ of $\mathrm{mKRB}$, or with mKRB-containing diluted inhibitors, $30 \mathrm{~min}$ before the ATP release test, except for $300 \mu \mathrm{M}$ O-ATP (in mKRB) that was preincubated for $120 \mathrm{~min}$. Inhibitors did not affect per se the total ATP cell content and the luciferase activity. The toxins were diluted in $25 \mu \mathrm{l}$ of mKRB and added to the wells at the zero time. The release of ATP due to mechanical effect was checked by adding $25 \mu \mathrm{l}$ of mKRB in control wells. During the pre-incubation and incubation time, cell plates were placed in incubator at $37^{\circ} \mathrm{C}, 5 \% \mathrm{CO}_{2}$. At different time points, the contents of at least three wells were collected and conserved on ice. Released ATP was determined with the ATPlite luciferase assay (Perkin-Elmer, Monza, Italy). Briefly, the supernatant of control and intoxicated samples were collected in a white 96-well plate, and the mammalian lysis solution, included in the assay kit, was added. The plate was shaken for $5 \mathrm{~min}$ in an orbital shaker at 700 r.p.m., RT. ATP substrate solution was added and a 5 -min shake was performed, protected from light. After $10 \mathrm{~min}$, the luminescence was measured using the Fluoroskan Ascent FL (Thermo Electron Corporation, Milan, Italy). The ATP concentration was calculated from a calibration curve obtained using ATP standard solutions.

Calcium Imaging. Cells plated on coverslips (24-mm diameter in six-well plates, $200000 \mathrm{cells} /$ well), were loaded with $9 \mu \mathrm{M}$ Fura-2/AM at $37^{\circ} \mathrm{C}$ for $20 \mathrm{~min}$, in $1 \mathrm{ml}$ of mKRB containing $0.04 \%$ pluronic F-127 (Molecular Probes, Inc., Eugene, OR, USA). To prevent fura-2 leakage and sequestration, sulfinpyrazone $(200 \mu \mathrm{M})$ was present throughout the loading procedure. Then, $1 \mathrm{ml}$ of mKRB was added to the mixture and cells were incubated for another $10 \mathrm{~min}$ at $37^{\circ} \mathrm{C}$. Subsequently, coverslips were washed with mKRB, mounted on a thermostatted chamber (Medical System Corp., New York, NY, USA) at $37^{\circ} \mathrm{C}$, and placed on an inverted microscope (Zeiss, Milan, Italy, Axiovert 100 TV) equipped for single cell fluorescence measurements and imaging analysis (TILL Photonics, Martinsried, Germany). Where indicated, a $\mathrm{Ca}^{2+}$-free EGTA $(200 \mu \mathrm{M})$-containing medium was used. The sample was alternatively illuminated $(t=200 \mathrm{~ms})$ by monochromatic light (at 340 and $380 \mathrm{~nm}$ wavelengths), every second for 10-20 min after toxin exposure, through a $\times 40$ oil immersion objective $(N A=1.30$; Zeiss). The emitted fluorescence was passed through a dichroic beam splitter (455DRPL), filtered at 505-530 nm (Omega Optical and Chroma Technologies, Brattleboro, VT, USA) and captured by a cooled CCD camera (Imago, TILL Photonics). For presentation, the ratios (F340/F380) of cells were off-line normalized to the resting value measured within the first minute of the experiment.

Fluorescence microscopy. Cells were plated on coverslips, and treated for $2 \mathrm{~h}$ with collagen (BD Biosciences, Milan, Italy). Before experiments, cells were incubated for $15 \mathrm{~min}$, at $37^{\circ} \mathrm{C}$, with $1 \mu \mathrm{M}$ calcein-AM (Life Technologies) diluted in
mKRB and then washed three times with mKRB. Alternatively, cells were incubated with annexin-V-fluorescein and propidium iodide (Annexin-V-FLUOS Staining Kit, Roche, Milan, Italy) in the incubation buffer provided with the kit. Effect of intoxication with Mt-II was observed with a wide-field inverted microscope (Leica Microsystems, Milan, Italy, DMI6000B) equipped with a DFC300FXR2 digital camera. Images were acquired sequentially with a FITC and Texas Red filter set (Chroma Technology Corp., Olking, Germany). The acquisition software was LAS AF6000 (Leica Microsystems).

\section{Conflict of Interest}

The authors declare no conflict of interest.

Acknowledgements. We thank P Caccin, I Zornetta and ML Massimino for helpful comments and suggestions. This work was supported by University of Padova Progetto Strategico 'An in vivo approach to the physiopathology of signal transduction' to $\mathrm{CM}$, a fellowship of the Fondazione CARIPARO to JF and the ICGEB-CRP Program (grant COS-08-03) to BL and JG.

1. Gutiérrez JM, Theakston RD, Warrell DA. Confronting the neglected problem of snake bite envenoming: the need for a global partnership. PLoS Med 2006; 3: e150.

2. Gutiérrez JM, Rucavado A, Chaves F, Díaz C, Escalante T. Experimental pathology of local tissue damage induced by Bothrops asper snake venom. Toxicon 2009; 54: 958-975.

3. Gutiérrez JM, Escalante T, Rucavado A. Experimental pathophysiology of systemic alterations induced by Bothrops asper snake venom. Toxicon 2009; 54: 976-987.

4. Hernández R, Cabalceta $C$, Saravia-Otten P, Chaves A, Gutiérrez JM, Rucavado A. Poor regenerative outcome after skeletal muscle necrosis induced by Bothrops asper venom: alterations in microvasculature and nerves. PLOS One 2011; 6: e19834.

5. Lomonte $B$, Angulo $Y$, Calderón L. An overview of lysine-49 phospholipase $A_{2}$ myotoxins from crotalid snake venoms and their structural determinants of myotoxic action. Toxicon 2003; 42: 885-901.

6. Fletcher JE, Hubert M, Wieland SJ, Gong QH, Jiang MS. Similarities and differences in mechanisms of cardiotoxins, melittin and other myotoxins. Toxicon 1996; 34: 1301-1311.

7. Wu WG, Tjong SC, Wu PL, Kuo JH, Wu K. Role of heparan sulfates and glycosphingolipids in the pore formation of basic polypeptides of cobra cardiotoxin. Adv Exp Med Biol 2010; 677: $143-149$.

8. Montecucco C, Gutiérrez JM, Lomonte B. Cellular pathology induced by snake venom phospholipase $A_{2}$ myotoxins and neurotoxins: common aspects of their mechanisms of action. Cell Mol Life Sci 2008; 65: 2897-2912.

9. Angulo $Y$, Lomonte B. Biochemistry and toxicology of toxins purified from the venom of the snake Bothrops asper. Toxicon 2009; 54: 949-957.

10. Harris JB. Myotoxic phospholipases $\mathrm{A}_{2}$ and the regeneration of skeletal muscles. Toxicon 2003; 42: 933-945.

11. Ciciliot S, Schiaffino S. Regeneration of mammalian skeletal muscle. Basic mechanisms and clinical implications. Curr Pharm Des 2010; 16: 906-914.

12. Cintra-Francischinelli M, Caccin $P$, Chiavegato A, Pizzo P, Carmignoto G, Angulo $Y$ et al. Bothrops snake myotoxins induce a large efflux of ATP and potassium with spreading of cell damage and pain. Proc Natl Acad Sci USA 2010; 107: 14140-14145.

13. Ferrari D, Chiozzi P, Falzoni S, Dal Susino M, Collo G, Buell G et al. ATP-mediated cytotoxicity in microglial cells. Neuropharmacology 1997; 36: 1295-1301.

14. Murgia M, Pizzo P, Steinberg TH, Di Virgilio F. Characterization of the cytotoxic effect of extracellular ATP in J774 mouse macrophages. Biochem J 1992; 288 (Pt 3): 897-901.

15. Praetorius HA, Leipziger J. ATP release from non-excitable cells. Purinergic Signal 2009; 5: 433-446.

16. Corriden R, Insel PA. Basal release of ATP: an autocrine-paracrine mechanism for cell regulation. Sci Signal 2010; 3, re1.

17. Sabirov RZ, Okada Y. The maxi-anion channel: a classical channel playing novel roles through an unidentified molecular entity. J Physiol Sci 2009; 59: 3-21.

18. Okada Y, Sato K, Numata T. Pathophysiology and puzzles of the volume-sensitive outwardly rectifying anion channel. J Physiol 2009; 587(Pt 10): 2141-2149.

19. Volonté C, D'Ambrosi N. Membrane compartments and purinergic signaling: the purinome, a complex interplay among ligands, degrading enzymes, receptors and transporters. FEBS J 2009; 276: 318-329.

20. Bok K, Prikhodko VG, Green KY, Sosnovtsev SV. Apoptosis in murine norovirus-infected RAW264.7 cells is associated with downregulation of survivin. J Virol 2009; 83: 3647-3656.

21. Aird SD. Ophidian envenomation strategies and the role of purines. Toxicon 2002; 40: 335-393.

22. Zuliani JP, Gutiérrez JM, Casais e Silva LL, Coccuzzo Sampaio S, Lomonte B, Pereira Teixeira CeF. Activation of cellular functions in macrophages by venom secretory Asp-49 and Lys-49 phospholipases $A_{2}$. Toxicon 2005; 46: 523-532.

23. Tidball JG, Wehling-Henricks $M$. Macrophages promote muscle membrane repair and muscle fibre growth and regeneration during modified muscle loading in mice in vivo. $J$ Physiol 2007; 578(Pt 1): 327-336. 
24. Lomonte B, Tarkowski A, Hanson LA. Broad cytolytic specificity of myotoxin II, a lysine-49 phospholipase $A_{2}$ of Bothrops asper snake venom. Toxicon 1994; 32: 1359-1369.

25. Leiguez E, Zuliani JP, Cianciarullo AM, Fernandes CM, Gutiérrez JM, Teixeira C. A group

IIA-secreted phospholipase $A_{2}$ from snake venom induces lipid body formation in macrophages: the roles of intracellular phospholipases $A_{2}$ and distinct signaling pathways. J Leukoc Biol 2011; 90: 155-166.

26. Näreoja K, Näsman J. Selective targeting of G-protein-coupled receptor subtypes with venom peptides. Acta Physiol 2011.

27. Murakami MT, Arruda EZ, Melo PA, Martinez AB, Calil-Eliás S, Tomaz MA et al. Inhibition of myotoxic activity of Bothrops asper myotoxin II by the anti-trypanosomal drug suramin. $\mathrm{J} \mathrm{Mol} \mathrm{Biol} \mathrm{2005;} \mathrm{350:} \mathrm{416-426.}$

28. Huang YA, Stone LM, Pereira E, Yang R, Kinnamon JC, Dvoryanchikov G et al. Knocking Out P2X Receptors reduces transmitter secretion in taste buds. J Neurosci 2011; 31 13654-13661.

29. Jacques-Silva MC, Correa-Medina M, Cabrera O, Rodriguez-Diaz R, Makeeva N, Fachado $A$ et al. ATP-gated $P 2 \times 3$ receptors constitute a positive autocrine signal for insulin release in the human pancreatic beta cell. Proc Natl Acad Sci USA 2010; 107: 6465-6470.

30. Kronlage M, Song J, Sorokin L, Isfort K, Schwerdtle T, Leipziger J et al. Autocrine purinergic receptor signaling is essential for macrophage chemotaxis. Sci Signal 2010; 3 , ra55.

31. Vylkova S, Sun JN, Edgerton M. The role of released ATP in killing Candida albicans and other extracellular microbial pathogens by cationic peptides. Purinergic Signal 2007; 3 : 91-97.

32. Skals M, Jorgensen NR, Leipziger J, Praetorius HA. Alpha-hemolysin from Escherichia coli uses endogenous amplification through $\mathrm{P} 2 \mathrm{X}$ receptor activation to induce hemolysis. Proc Natl Acad Sci USA 2009; 106: 4030-4035.

33. Skals M, Jensen UB, Ousingsawat J, Kunzelmann K, Leipziger J, Praetorius HA Escherichia coli alpha-hemolysin triggers shrinkage of erythrocytes via $\mathrm{K}(\mathrm{Ca}) 3.1$ and
TMEM16A channels with subsequent phosphatidylserine exposure. J Biol Chem 2010; 285: 15557-15565.

34. Díaz C, León G, Rucavado A, Rojas N, Schroit AJ, Gutiérrez JM. Modulation of the susceptibility of human erythrocytes to snake venom myotoxic phospholipases $\mathrm{A}_{2}$ : role of negatively charged phospholipids as potential membrane binding sites. Arch Biochem Biophys 2001; 391: 56-64.

35. Konshina AG, Boldyrev IA, Utkin YN, Omel'kov AV, Efremov RG. Snake cytotoxins bind to membranes via interactions with phosphatidylserine head groups of lipids. PLOS One 2011; 6: e19064.

36. Rangel J, Quesada O, Gutiérrez JM, Angulo Y, Lomonte B. Membrane cholesterol modulates the cytolytic mechanism of myotoxin II, a Lys 49 phospholipase $A_{2}$ homologue from the venom of Bothrops asper. Cell Biochem Funct 2011; 29: 365-370.

37. Junger WG. Immune cell regulation by autocrine purinergic signalling. Nat Rev Immuno 2011; 11: 201-212.

38. Cathcart MK. Signal-activated phospholipase regulation of leukocyte chemotaxis. J Lipid Res 2009; 50, Suppl S231-S236.

39. Qu Y, Dubyak GR. P2 $\times 7$ receptors regulate multiple types of membrane trafficking responses and non-classical secretion pathways. Purinergic Signal 2009; 5: 163-173.

40. Séror C, Melki MT, Subra F, Raza SQ, Bras M, Saïdi H et al. Extracellular ATP acts on P2Y2 purinergic receptors to facilitate HIV-1 infection. J Exp Med 2011; 208: 1823-1834.

Cell Death and Disease is an open-access journal published by Nature Publishing Group. This work is licensed under the Creative Commons Attribution-NonCommercialShare Alike 3.0 Unported License. To view a copy of this license, visit http://creativecommons.org/licenses/by-nc-sa/3.0/

\section{Supplementary Information accompanies the paper on Cell Death and Disease website (http://www.nature.com/cddis)}

